$\xi=-1$

\title{
Identity-Based Data integrity checking in public cloud with bilinear pairings
}

\author{
B.B.V. Satya Vara Prasad ${ }^{*}$, Ch. Hari Kishan ${ }^{2}$, S.P. Praveen ${ }^{3}$, Ch. Mani Teja ${ }^{4}$ \\ ${ }^{1}$ Assistant Professor, Department of Electronics \& Computer Engineering, KLEF, AP, India \\ ${ }^{2}$ Associate Professor,Department of Computer Science Engineering,SACET,Chirala,AP,India \\ ${ }^{3}$ B.Tech Student, Department of Electronics \& Computer Engineering, KLEF, AP , India \\ ${ }^{4}$ B.Tech Student, Department of Electronics \& Computer Engineering, KLEF,AP ,India \\ Green Fields, Vaddeswaram, Guntur, Andhra Pradesh 522502. \\ *Email: bbhanuprasad@kluniversity.in
}

\begin{abstract}
A consistently expanding number of clients should need to stock their information in servers that are public close by the quick headway of cloud computing. Novel security issues must be grasped remembering the true objective to empower more number of customers to process their information in broad daylight. Exactly when the user is confined to get to PCS, then they will assign its intermediary to process their information and transfer them. However remote information trustworthiness inspection is in like manner a basic security issue in broad daylight distributed storing. This impacts the clients to examine in case their outsourced data are held in reserve, set up without copying the complete data. In the previous paper Diffie Hellman algorithm is replaced with elliptic curve cryptography based key exchange algorithm. To compete with Diffie Hellman the algorithm is not so secure. As of the safety complications, we suggest a different midway settled information transporting and remote data dependability inspection prototype in character dependent open key cryptography :character based intermediary arranged information transferred what's more, remote data respectability checking transparently cloud (ID-ICBP) with Tate pairings which is better when compared to Diffie Hellman.. We provide the formal description, structure model, and security show up. By at that point, a solid ID-ICBP custom is completed utilizing the bilinear pairings. The suggested ID-ICBP convention is provably protected in context of the hardness of computational Diffie- Hellman issue. Our ID-ICBP custom is in like way convincing and adaptable. In light of the intriguing customer's support, the suggested ID-ICBP convention can recognize private remote information uprightness checking, appointed remote information respectability checking, and open remote information uprightness checking.
\end{abstract}

\section{Introduction}

Out in the open cloud state, most of the customers exchange their info to PCS and examine the remote info dependability by net. Right after the customer is an specific decision making, some even minded problems will occur. If the chief is linked through being incorporated into the business distortion then he will be behind the bars. In the midst of the period of inspection, the manager will be constrained to get to the outline recalling the true objective to screen against interest. Regardless, the head's true blue business will go ahead in the middle of the period of inspection. Bilinear pairings have been utilized to outline sharp conventions for such undertakings as one-cycle three-party key assention, identity based encryption, furthermore aggregate signatures. Appropriate bilinear pairings can be built from the Tate pairings for uniquely picked elliptic curves.

Short signatures: We present a short mark plot in view of the Diffie- Hellman suspicion on different elliptic curves. For basic safety parameters, the length of the signature is about a large portion of that of the signature of a DSA with a comparable security The short signature plot is intended for frameworks where marks are composed in by a person or being transmitted over a lowtransmission capacity channel. We review various properties of our mark plan, for example, signature total and batch confirmation.

Correctly once a broad information is prepared, who can authorize him to process these information? On the off chance that these data can't be dealt with without a moment to spare, the director will confront the loss of cash related premium. To divert the happening of this case, the overseer needs to choose the center individual to process its information, for instance, his secretary. Nevertheless, the head will have a hard time trusting others can play out the remote information uprightness checking .Open inspection will realize some risk of discharging the security. For illustration, the set away data volume be able to be recognized by means of the poisonous authenticators. Right after the exchanged data volume is characterized, private remote data trustworthiness inspection is very important. Regardless of the way that the secretary can process besides, exchange the data for the manager, in spite of all that he can't examine the main's remote information genuineness excluding he is appointed via the superior. We call the administrator as the go-between of the executive.

In PKI (open key framework), remote data decency looking at tradition will show the presentation organization. Right when the administrator assigns some components to show out the remote information dependability inspection, it will achieve noteworthy outlays since the evaluator will examine the revelation after it checks the remote data morality. In PKI, the broad outlays start 
from the generous confirmation affirmation, supports age, conveyance, revocation, reclamation. Transparently circulated figuring, the end devices may have low count constrain, for instance, PDA, iPad. Identity based open key cryptography can discard the jumbled support organization. Remembering the true objective to grow the capability, character based intermediary arranged information exchanging and remote information trustworthiness inspection is all the more appealing. Subsequently, it will be outstandingly critical to think about the ID-PUIC tradition.

\section{Related Work}

Cloud computing is ending up progressively prominent. Innumerable are outsourced to the cloud by data proprietors moved to get to the broad scale figuring properties and budgetary venture stores. To secure data insurance, the delicate data should be encoded by the data proprietor before outsourcing, which makes the standard and capable plaintext catchphrase look strategy vain. So how to design a successful, in the two sections of precision and profitability, available encryption plot over mixed cloud data is a particularly troublesome errand. In this paper, out of the blue, we propose a down to earth, proficient, and adaptable accessible encryption conspire which underpins both multi-catchphrase positioned pursuit and parallel hunt. To help multi-catchphrase pursuit and result pertinence positioning, we embrace Vector Space Model (VSM) to construct the accessible file to accomplish exact list items. To enhance look proficiency, we outline a tree-based list structure which underpins parallel inquiry to exploit the intense processing limit and assets of the cloud server. With our outlined parallel hunt calculation, the pursuit proficiency is very much made strides. We propose two secure accessible encryption strategies to encounter distinctive protection requirements in two danger models. Broad investigations on this current truth dataset support our examination and demonstrate that our projected arrangement is extremely proficient and powerful in supporting multi-watchword positioned parallel pursuits. cloud storing is currently a burning study point in data innovation. In cloud storing, data safekeeping assets, for example, information secure, trustworthiness and openness turn out to be more and more imperative in several commercial applications. As of late, numerous Provable Data Possession (PDP)schemes are projected to ensure information trustworthiness. At times, it requires to assign the remote data control inspection errand to some intermediary. Nevertheless, these PDP designs won't be protected as the delegate stores some state information in storage servers. Thus, in this paper, we propose a productive common irrefutable provable information ownership plot, that practices Diffie-Hellman mutual key to improve the homomorphism validation. The verifier here is stateless and restricted of the circulated stockpiling advantage. It is huge that the presented plot is especially beneficial differentiated and the past PDP designs, since the bilinear activity isn't required. In the previous papers they mentioned about 1st phase and 2nd phase challenges. But here no challenge is possible because it is so secure.

\section{Framework}

The suggested solid ID-ICBP protocol covers four procedures: Arrangement, Citation, Proxy-key triggering, TagGen and Proof With a specific end goal to reveal the nature of our improvement, the solid convention's strategy is delineated.

\section{Solid ID-ICBP Protocol:-}

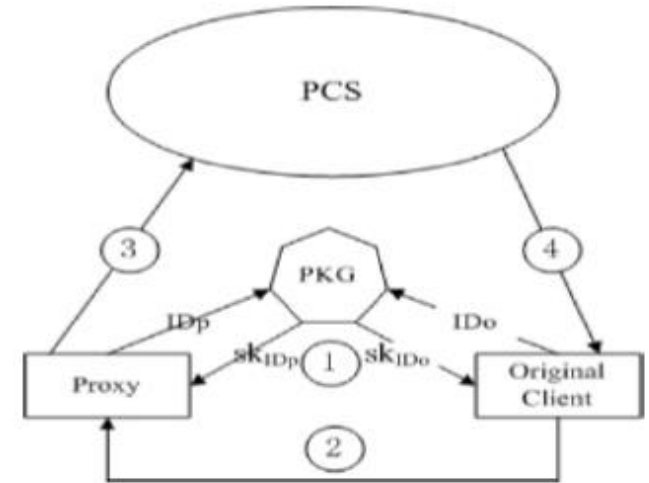

Fig:1: Architecture of our ID-ICBP protocol.

To begin with, Setup is performed and the framework parameters are created. In view of the produced framework parameters, alternate strategies are performed. It can be described as (1) During the stage Citation, when the substance's character becomes the input, KGC produces the private key for the entity. Particularly, production of the private keys for customer and for the proxy. (2) During the stage of Proxy-key generating, the customer makes the license and enables the proxy to produce the key. (3) During Tag Generation, once the information hunk is input, the proxy creates the block's label and then uploads the block tag couples sets to PCS. (4) Finally, during Proof the original customer S associates with PCS. Over the association, $\mathrm{S}$ checks his remote information respectability. Ensuing the protocol's engineering, we stretch the solid development Deprived of damage of simplification, assume the proxy intends to transfer the record.

- Setup: Assume the two groups C1, C2 and bilinear pairings is assumed to be b. A similar request is for both of the $\mathrm{C} 1$ and $\mathrm{C} 2$. Let e be the generator e of the gathering $\mathrm{C} 1$.

Cryptographic hash functions derived are:

$\mathrm{H}:\{0,1\} * \rightarrow \mathrm{X} * \mathrm{r}, \mathrm{h}: \mathrm{X} * \mathrm{r} \times\{0,1\} * \rightarrow \mathrm{C} 1$

Assume a pseudo-irregular capacity $\mathrm{g}$ and a pseudo-arbitrary stage $\pi$. The above two capacities $g$ and $\pi$ are characterized beneath:

$\mathrm{g}: \mathrm{X} * \mathrm{r} \times\{1,2, \mathrm{n}\} \rightarrow \mathrm{X} * \mathrm{r} \pi: \mathrm{X} * \mathrm{r} \times\{1,2, \mathrm{n}\} \rightarrow\{1,2, \mathrm{n}\}$

KGC produces its top secret key y where $y \in X * r$. At that point, it processes $\mathrm{Y}=\mathrm{gy}$. The limitations $\{\mathrm{C} 1, \mathrm{C} 2, \mathrm{~b}, \mathrm{r}, \mathrm{e}, \mathrm{Y}, \mathrm{H}, \mathrm{h}, \mathrm{f}, \pi\}$ are ended open. The top secret key y is with the KGC.

Extract: Say the original customer's character I Do, KGC prefers an arbitrary Go $\in \mathrm{X} * \mathrm{r}$ and figures (Go, $\sigma \mathrm{o})$.

$\mathrm{Go}=$ gro, $\sigma \mathrm{o}=\mathrm{ro}+\mathrm{yH}(\mathrm{I}$ Do, Go $) \bmod \mathrm{r}$

At that point, $\mathrm{KGC}$ sends sk I Do $=(\mathrm{Go}, \sigma o)$ to the original customer by the secure channel. Give sk I A chance to do be the first customer's private key. The first customer checks skIDo's rightness by confirming the accompanying condition.

$\mathrm{g} \sigma \mathrm{o}=\mathrm{GoY} \mathrm{H}(\mathrm{I}$ Do,Go $)$.

\section{Proxy-key generation:}

So as to create the intermediary key, the first customer I Do has to associate through the proxy I Dp.

i Do makes the permit $m \omega$ as per its necessities. The intermediary i Dp can't develop and transfer the first customer i Do's information except it fulfils $m \omega$.The $i$ Do pick an irregular $q 1 \in X * r$ and figures $m \omega$ 's mark.

$\mathrm{G} 1=\operatorname{gr} 1, \sigma 1=\mathrm{q} 1+\sigma \mathrm{oH}(\mathrm{m} \omega, \mathrm{G} 1) \bmod \mathrm{r}$

The i Do sends the warrant-signature $(\mathrm{m} \omega, \mathrm{R} 1, \sigma)$ and Ro to i Dp and PCS.

The i Dp checks the validity of ( $m \omega, \mathrm{R} 1, \sigma, \mathrm{Ro})$ with respect to the following equation.

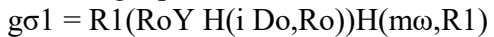

On the off chance that the confirmation is not successful, the proxy will reject that one and recommends i Do; else, it figures the key generation: $\sigma o=\sigma+\sigma p H(m \omega, R 1)$ The secret key $\sigma o$ is kept secret by the proxy.In the meantime, Rp is sent to iDo by iDp . 
- TagGen: Once the i Dp fulfils the warrant m $\omega$, i Dp has to enable iDo develops its information. Assume the original customer's plaintext record is ^A. By utilizing the symmetric encryption, ${ }^{\wedge} \mathrm{A}$ is encoded to the ciphertext after that it is uploaded to PCS. In view of the measure of $\mathrm{F}$, the proxy $\mathrm{i}$ Dp parts $\mathrm{F}$ into $\mathrm{n}$ blocks, Hi means the $i$ th block of $\mathrm{F}$ and $\mathrm{Hi} \in \mathrm{X} * \mathrm{r}$. Ni contains the $\mathrm{i}$ th block Hi 's name and all its assets .

The intermediary computes $\mathrm{u}=\mathrm{h}(\mathrm{n}+1$, iD0).

At that point, for $1 \leq \mathrm{i} \leq \mathrm{n}$, the intermediary plays out the accompanying strategies.

The proxy registers $\mathrm{Pi}=($ howdy, $\mathrm{Ni}) \mathrm{uFi}$ ) $\sigma$ by utilizing the intermediary key $\sigma ; 2$ ) The proxy output block Hi 's label Pi . Finally, the proxy contracts all the piece label sets $\{(\mathrm{Hi}, \mathrm{Pi}), 1 \leq \mathrm{I} \leq \mathrm{n}\}$ and transfers them to PCS.

At the point when PCS gets m $\omega$ 's short signature $(\mathrm{m} \omega, \mathrm{G} 1, \sigma 1)$ and Go, it checks ( $m \omega, \mathrm{Gl}, \sigma 1$ 's) legitimacy by confirming . e $\sigma 1=\mathrm{G} 1(\mathrm{GoY} H(\mathrm{I}$ Do,Go $)) \mathrm{H}(\mathrm{m} \omega, \mathrm{R} 1)$ holds.

On the off chance that it will hold, PCS acknowledges m $\omega$; else, it educates I Do. While getting the square label sets $\{(\mathrm{Fi} \mathrm{Ti}), 1 \leq \mathrm{I} \leq$ $\mathrm{n}\}$, PCS has to check if the I Dp fulfills $m \omega$. In the event that it will hold, PCS acknowledges and store it; generally, PCS declines to acknowledge them.

\section{Experiment Results}

We give the count and correspondence overhead of our projected ID-ICBP tradition. Meanwhile, we complete the model of our IDICBP tradition and survey its shot price.At that point, we give the flexibility of remote information respectability inspection in the stage Proof in the ID-ICBP tradition. At last, we differentiate our ID-ICBP tradition and the other up to-date remote data uprightness inspection traditions.
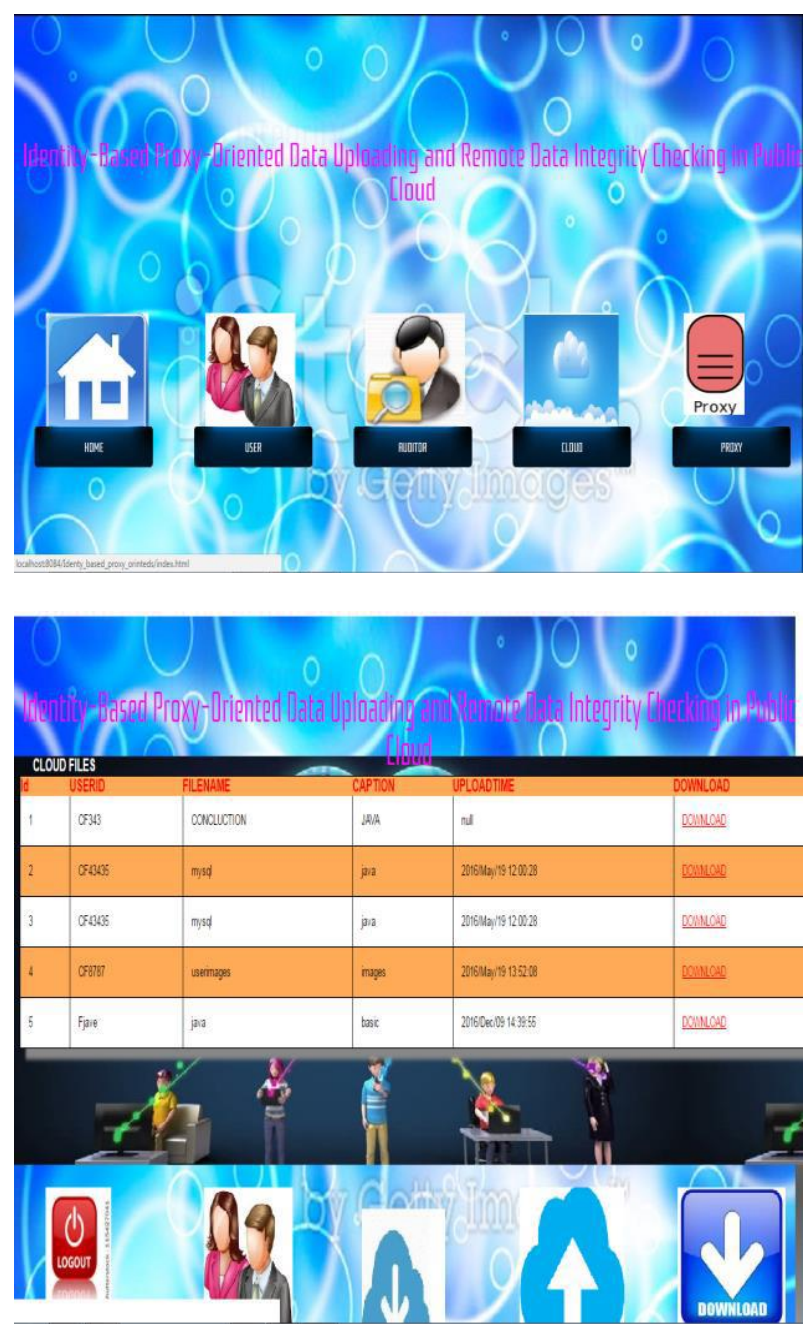

\section{Conclusion}

Propelled by means of the consumer requirements, this paper offers a fresh safety design of ID-ICBP in wide-ranging public cloud. This paper validates ID-ICBP's outline model and security model. On that idea, the main solid ID-ICBP resolution is composed by employing the bilinear pairings approach. The solid ID-ICBP resolution stands provably protected by employing the official safety confirmation and efficiency investigation. Yet again, the projected ID-ICBP resolution can equally recognize private remote information uprightness checking, appointed remote info honesty inspection and open remote info trustworthiness inspection for first customer's approval.

\section{Acknowledgement}

We express our sincere thankfulness to our project guide Mr.B.B.V.Satya Vara Prasad for his successful guidance to our project.Without his help, it would be a tough job to accomplish. We thank our guide for his encouragement throughout out period of work .We also thank our Head of the Department (ECSE) Dr.K.Raghava Rao for providing us all the necessary facilities.

\section{References}

[1] Z. Fu, X. Sun, Q. Liu, L. Zhou, and J. Shu, "Achieving efficient cloud search services: Multi-keyword ranked search over encrypted cloud data supporting parallel computing," IEICE Trans. Commun., vol. E98-B, no. 1, pp. 190-200, 2015.

[2] Y. Ren, J. Shen, J. Wang, J. Han, and S. Lee, "Mutual verifiable provable data auditing in public cloud storage," J. Internet Technol., vol. 16, no. 2, pp. 317-323, 2015.

[3] M. Mambo, K. Usuda, and E. Okamoto, "Proxy signatures for delegating signing operation," in Proc. CCS, 1996, pp. 48-57.

[4] E.-J. Yoon, Y. Choi, and C. Kim, "New ID-based proxy signature scheme with message recovery," in Grid and Pervasive Computing (Lecture Notes in Computer Science), vol. 7861. Berlin, Germany: Springer- Verlag, 2013, pp. 945-951.

[5] B.-C. Chen and H.-T. Yeh, "Secure proxy signature schemes from the weil pairing," J. Supercomput., vol. 65 , no. 2 , pp. 496 506, 2013.

[6] X. Liu, J. Ma, J. Xiong, T. Zhang, and Q. Li, "Personal health records integrity verification using attribute based proxy signature in cloud computing," in Internet and Distributed Computing Systems (Lecture Notes in Computer Science), vol. 8223. Berlin, Germany: Springer- Verlag, 2013, pp. 238-251.

[7] H. Guo, Z. Zhang, and J. Zhang, "Proxy re-encryption with unforgeable re-encryption keys," in Cryptology and Network Security (Lecture Notes in Computer Science), vol. 8813. Berlin, Germany: Springer-Verlag, 2014, pp. 20-33.

[8] E. Kirshanova, "Proxy re-encryption from lattices," in PublicKey Cryptography (Lecture Notes in Computer Science), vol. 8383. Berlin, Germany: Springer-Verlag, 2014, pp. 77-94.

[9] P. Xu, H. Chen, D. Zou, and H. Jin, "Fine-grained and heterogeneous proxy re-encryption for secure cloud storage," Chin. Sci. Bull., vol. 59, no. 32, pp. 4201-4209, 2014.

[10] S. Ohata, Y. Kawai, T. Matsuda, G. Hanaoka, and K. Matsuura, "Re-encryption verifiability: How to detect malicious activities of a proxy in proxy re-encryption," in Proc. CT-RSA Conf., vol. 9048. 2015, pp. 410-428. 